\title{
Variaciones adaptativas en la talla de la megafauna bentónica de fondos blandos tropicales en función de parámetros bióticos y abióticos
}

\author{
Luis A. Gomez-Lemos ${ }^{1} \&$ Camilo B. García ${ }^{2 *}$ \\ 1. Griffith University, School of Environment, Nathan campus, Building N13, Brisbane, Australia; \\ luis.gomezlemos@griffithuni.edu.au \\ 2. Universidad Nacional de Colombia, Departamento de Biología, Av. Cra. 30 \# 45-03, Bogotá D.C., Colombia; \\ cbgarciar@unal.edu.co \\ * Correspondencia
}

Recibido 08-VII-2016. Corregido 10-II-2017. Aceptado 13-III-2017.

\begin{abstract}
Adaptive variation in size of tropical soft bottom benthic megafauna related to biotic and abiotic factors. Understanding and predicting adaptations in body size of megabenthic invertebrates remains a major challenge in marine macroecology. This study was conducted in order to investigate size variations of benthic megafauna in the tropics and to identify the effect of biotic and abiotic factors that may produce changes to these organisms, testing unresolved hypothesis and paradigms of deep sea ecology from subtropical and temperate areas. The study area covered the continental shelf of the Colombian Caribbean. The samples were collected during 1998, 2001 and 2005, using semi-globe demersal net for a water depth of 10 to $500 \mathrm{~m}$. The most common species were selected for further study: Eudolium crosseanum, Cosmioconcha nitens, Nuculana acuta (mollusks), Astropecten alligator, Brissopsis atlantica, B. elongata (equinoderms), Anasimus latus, Chasmocarcinus cylindricus and Achelous spinicarpus (crustaceans). Generalized Additive Models were used to detect significant changes in size and to infer the effects of biotic and environmental factors on organisms' size. The dependent variable was size and the predicting model variables were depth, temperature, intraspecific density, interspecific density, richness, latitude, and longitude. A total of 7000 individuals were measured. Six species showed an increase in body size towards deeper and colder sites. These species inhabit shallow and deep environments that exceed a variation in temperature of $10{ }^{\circ} \mathrm{C}$. There was a remarkable size reduction in areas affected by the Magdalena River, possibly due to major physicochemical changes caused by the river. This region has the lowest planktonic primary productivity within the study area. An increase in sizes was observed north of the Magdalena River $\left(74^{\circ}-71^{\circ} \mathrm{W} \& 11^{\circ}-13^{\circ} \mathrm{N}\right)$, which may be attributable to the coastal upwelling occurring in this part of Colombia. The relationship between the density of benthic organisms and size was not clear. However, five species showed an inverse relation with intraspecific density and three with interspecific density. Temperature and depth were the variables that best explained the variations in size. Most of the studied species showed an increase in body size when temperature dropped along the bathymetric range. The trend of increasing size in deeper zones is contrary to the prediction of the optimal size theoretical model (but consistent with recent studies), which indicates a reduction in organisms' size in the deep sea, due to food limitation with increasing depth. It is possible that this increase in size is an adaptation to maximize energy, which is frequently observed in the coldest habitats of several species. Future studies in Caribbean should examine variations in size of benthic megafauna towards deeper zones (more than $500 \mathrm{~m}$ ), were temperature is less variable and then other factors can play a more important role determining the size of these organisms. Rev. Biol. Trop. 65 (3): 1002-1021. Epub 2017 September 01.
\end{abstract}

Key words: body size, predictor variables, benthic megafauna, tropical soft-bottoms, Colombian Caribbean Sea.

Comúnmente se ha considerado que la característica más notoria de los invertebrados bentónicos (meiofauna, macrofauna y megafauna) de zonas profundas es la pequeña talla exhibida por la mayoría de especies, en comparación con su contraparte de aguas someras (Gage \& Tyler, 1991; Warwick \& Clarke, 1996; Rex et al., 2006). Este patrón fue detectado 
inicialmente en expediciones del siglo XIX (Rex \& Etter, 1998), pero estudios realizados en diferentes partes del planeta reportan resultados contradictoríos (Polloni, Haedrich, Rowe, \& Clifford, 1979; Pfannkuche, 1985; Lampitt, Billet \& Rice, 1986; Jensen, 1988; Tietje, Deming, Rowe, Macko, \& Wilke, 1989). Los modelos teóricos de talla óptima formulados por Sebens (1982; 1987), basados en experimentación con anémonas, predicen un decrecimiento del tamaño de los invertebrados marinos con la profundidad, debido a la disminución de alimento hacia zonas profundas del océano (Sanders \& Hessler, 1969; Parsons, Takahashi, \& Hargrave, 1977; Hoey, Degraer, \& Vincx, 2004). Contrario a dichos modelos, otros autores (Peters, 1983; Mahaut, Sibuet, \& Shirayama, 1995; Rex \& Etter, 1998; Rex, Etter, Clain, \& Hill, 1999, Berkenbusch, Probert, \& Nodder, 2011) sugieren que la menor disponibilidad de alimento con la profundidad, pueden favorecer tamaños grandes, ya que organismos de mayor talla son metabólicamente más eficientes por unidad de masa (Childress \& Thuesen, 1993; Olabarria \& Thurson, 2003; Rex et al., 2006).

La temperatura también tiene un papel importante en la determinación de las tallas. Pequeñas variaciones de temperatura entre hábitats pueden causar grandes diferencias en el tamaño corporal, en las tasas de crecimiento y en la distribución de frecuencia de tallas de las poblaciones. La talla de muchos poiquilotermos puede incrementarse experimentalmente manteniéndolos a bajas temperaturas. Además, los animales tienden a ser de mayor tamaño en las porciones más frías de su ámbito geográfico, posiblemente como una adaptación para conservar energía (Sebens, 1987).

En macroecología existe un creciente interés en la relación entre la talla, la riqueza de especies y la abundancia de individuos, pero la naturaleza e implicaciones de esta relación no han sido suficientemente estudiadas (Blackburn \& Gaston, 1994; Warwick \& Clarck, 1996; Durden, Bett, Jones, Huvenne, \& Ruhl., 2015). Para muchos animales terrestres existe una relación inversa entre abundancia y talla (May, 1988). Igualmente ocurre en zonas marinas someras, pero en zonas profundas del océano dicho patrón no es claro (Abele, 1974; Danovaro, Snelgrove, \& Tyler, 2014; Hardy, Smith \& Thurnherr, 2015). En términos generales, la relación entre talla y riqueza es mejor conocida en ambientes terrestres que en marinos (May, 1988; Webb, 2012).

La presente investigación se centró en la respuesta adaptativa de la talla de la megafauna bentónica frente a la riqueza de especies, a la abundancia de individuos y a otros factores en el gradiente de profundidad, ya que la información sobre estos tópicos es considerada insuficiente (Warwick \& Clarke, 1996; Rex \& Etter, 1998; Leduc, Pilditch, \& Nodder, 2016). Además, la mayoría de investigaciones en esta temática se han realizado en regiones subtropicales (Mcclain, Rex, \& Etter, 2009) y el comportamiento de las variables (como la temperatura) que influyen sobre el tamaño de estos organismos en ambientes tropicales podrían generar tendencias diferentes a las registradas en aguas subtropicales. El único antecedente existente para el área de estudio (Caribe colombiano entre 20 y $550 \mathrm{~m}$ de profundidad) indica que los crustáceos de los géneros Agononida, Munida y Munidopsis son más grandes a mayores profundidades (Fierro, 2004).

De acuerdo a lo anterior, este estudio tuvo como propósito determinar la influencia de variables bióticas (densidad intraespecífica, densidad interespecífica, número de especies o riqueza) y ambientales (profundidad, temperatura, latitud y longitud) que plausiblemente pueden afectar el tamaño corporal de la megafauna epibentónica de fondos blandos tropicales en el Caribe colombiano. Para el cumplimiento de estos objetivos, se seleccionaron especies con una amplia distribución batimétrica y geográfica en el área de estudio, $\mathrm{y}$, por medio de modelos aditivos generalizados (GAM, por sus siglas en inglés), se establecieron las tendencias de cambio de tamaño de los organismos en relación con las variables biológicas y ambientales escogidas. 


\section{MATERIAL Y MÉTODOS}

El área de estudio abarca los fondos blandos del mar Caribe colombiano entre 10 y 500 $\mathrm{m}$ de profundidad, desde la zona fronteriza con Panamá hasta el área limítrofe con Venezuela (Fig. 1). Dentro de esta área hay arrecifes coralinos y rocosos, praderas marinas y algales, y fondos blandos heterogéneos que constituyen el ecosistema más extenso (Invemar, 2002a). El Caribe colombiano puede ser dividido en dos categorías oceanográficas principales: la zona sur, desde el golfo de Urabá hasta la desembocadura del río Magdalena, que cuenta con atributos típicos de mares tropicales; y la zona norte, desde unos pocos kilómetros al este del río Magdalena hasta La Guajira (Fig. 1), que se caracteriza por condiciones oceanográficas similares a las de aguas subtropicales, con focos de surgencia, que la convierten en la zona más productiva del Caribe colombiano (Álvarez-León, Aguilera-Quiñones, AndradeAmaya, \& Novak, 1995).
La megafauna bentónica fue recolectada durante los años 1998, 2001 y 2005 en tres cruceros de investigación realizados por el Instituto de Investigaciones Marinas y Costeras, Invemar (Invemar, 2000, 2002b; Invemar \& Corpoguajira, 2006). Debido a que los muestreos fueron realizados en diferentes años, se asumió que los tamaños de los organismos no son afectados considerablemente por la variación estacional o interanual. Las muestras se recolectaron con una red demersal tipo semiglobo, con una relinga superior de $7.70 \mathrm{~m} \mathrm{e}$ inferior de $9.50 \mathrm{~m}$, y longitud total de $12.7 \mathrm{~m}$ (cuerpo de la red incluidas las alas: $9.10 \mathrm{~m} \mathrm{y}$ el copo: $3.60 \mathrm{~m}$ ). Los arrastres se realizaron durante 10 minutos, a tres nudos de velocidad (1.85 kilómetros por hora), en las isobatas de $10,20,50,70,150,300$ y $500 \mathrm{~m}$.

Con base en el Sistema de Información sobre Biodiversidad Marina (SIBM) del Invemar, se seleccionaron las especies mejor representadas (con amplia distribución geográfica

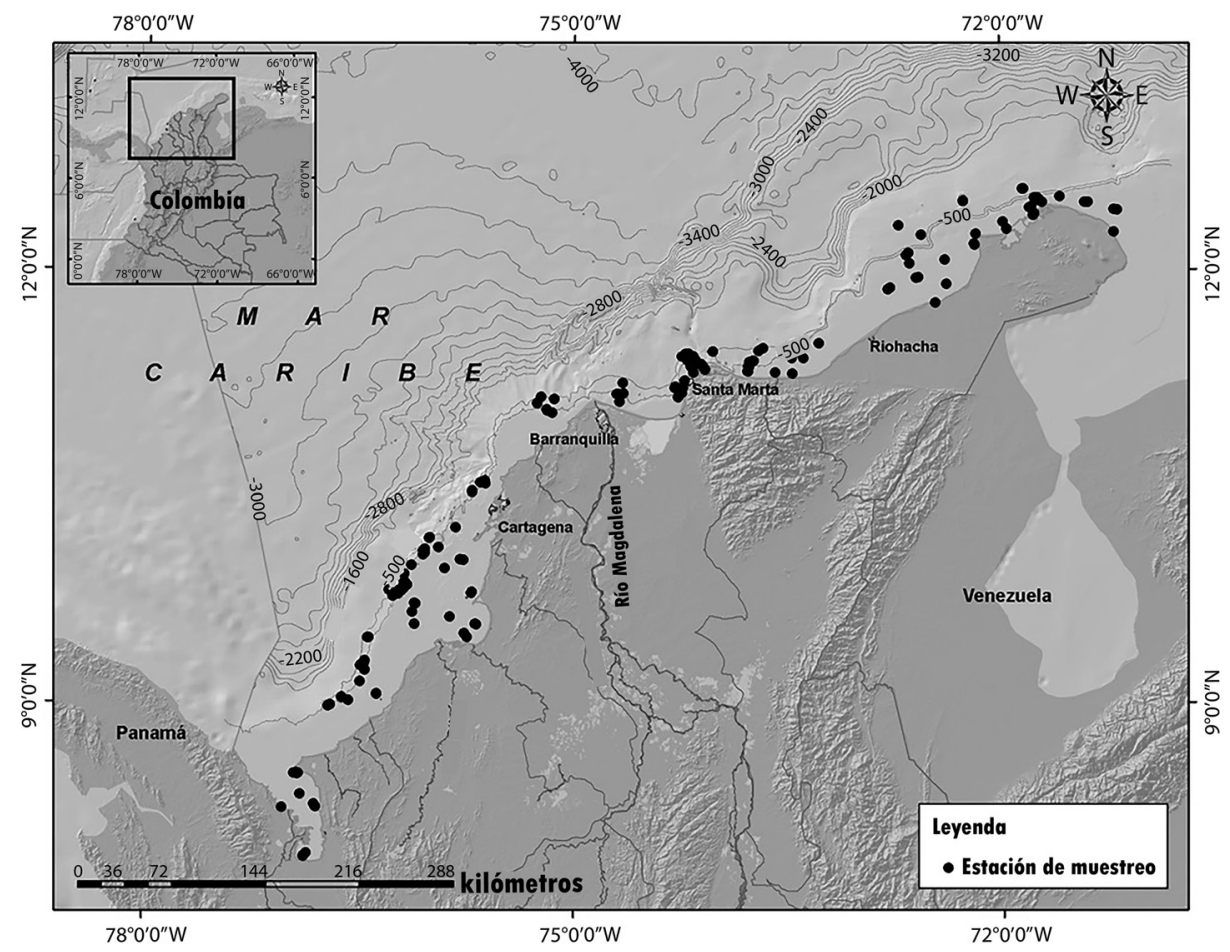

Fig. 1. Área de estudio con los sitios de recolecta de los ejemplares.

Fig. 1. Location of the study area showing the sampling sites. 
CUADRO 1

Resultados de la modelación GAM entre el tamaño de los ejemplares (mm) y las variables predictivas biológicas

TABLE 1

GAM results of the relationship between length $(\mathrm{mm})$ and biological predictor variables

\begin{tabular}{lccccccc}
\hline \multirow{2}{*}{ Especie } & \multirow{2}{*}{ Medida } & \multicolumn{2}{c}{ Riqueza } & \multicolumn{2}{c}{ Densidad Intrasp } & \multicolumn{2}{c}{ Densidad Intersp } \\
\cline { 3 - 8 } & & Dev (\%) & $\mathrm{P}$ & Dev (\%) & $\mathrm{P}$ & Dev (\%) & $\mathrm{P}$ \\
\hline Astropecten alligator & $\mathrm{R}$ & 6.685 & 0.000 & 11.553 & 0.000 & 5.640 & 0.000 \\
Anasimus latus & $\mathrm{LC}$ & 5.747 & 0.023 & 7.602 & 0.014 & 3.954 & 0.233 \\
Brissopsis atlantica & $\mathrm{LT}$ & 13.700 & 0.000 & 9.090 & 0.000 & 29.635 & 0.000 \\
Brissopsis elongata & $\mathrm{LT}$ & 42.458 & 0.000 & 81.481 & 0.000 & 30.769 & 0.000 \\
Chasmocarcinus cylindricus & $\mathrm{LC}$ & 9.956 & 0.048 & 38.847 & 0.329 & 37.000 & 0.000 \\
Cosmioconcha nitens & $\mathrm{AL}$ & 98.947 & 0.000 & 65.384 & 0.013 & 95.454 & 0.016 \\
Eudolium crosseanum & $\mathrm{AL}$ & 65.306 & 0.003 & 28.063 & 0.060 & 67.010 & 0.000 \\
Nuculana acuta & $\mathrm{L}$ & 10.039 & 0.000 & 13.139 & 0.000 & 7.190 & 0.000 \\
Achelous spinicarpus & $\mathrm{LC}$ & 15.203 & 0.000 & 31.766 & 0.000 & 21.439 & 0.000 \\
\hline
\end{tabular}

$\mathrm{La}(\mathrm{s})$ celda(s) resaltada(s) es una de las dos (o las dos) que mostro mayor asociación con la variación de las tallas de las diferentes especies. Dev: desviación explicada por el modelo. R: radio mayor. LC: largo de caparazón. LT: largo de la testa. AL: altura de la concha. L: largo de las valvas.

Cells in grey show the variable(s) that better explain the variation in length for each species.

y batimétrica) en el área de estudio: los moluscos Eudolium crosseanum, Cosmioconcha nitens (clase Gastropoda) y Nuculana acuta (clase Bivalvia); los equinodermos Astropecten alligator (clase Asteroidea), Brissopsis atlantica y Brissopsis elongata (clase Echinoidea); y los crustáceos decápodos Anasimus latus, Chasmocarcinus cylindricus y Achelous spinicarpus. A los gasterópodos se les determinó el alto y ancho de la concha; a los bivalvos el largo y alto de las valvas (Sturm, Pearce, \& Valdés, 2007); a los asteroideos el radio mayor y menor, y a los echinoideos el largo y ancho de la testa (Marcus, 1983). A los crustáceos decápodos se midió la longitud y el ancho del caparazón (Escobar-Briones \& Alvarez, 2002). Las medidas finalmente usadas en el análisis se reseñan en el cuadro 1. Para realizar este estudio se determinaron las dimensiones de 7000 ejemplares de las nueve especies estudiadas: $E$. Crosseanum (146), C. nitens (285), N. acuta (2507), A. alligator (428), B. atlantica (308), B. elongata (113), A. latus (249), C. cylindricus (1149), A. spinicarpus (1815).

La densidad interespecífica e intraespecífica por área barrida se calculó con las matrices de abundancia de cada campaña de muestreo. La densidad interespecífica es el número total de ejemplares de todas las especies presentes en cada estación de muestreo y la intraespecífica indica únicamente la densidad de cada especie objeto de estudio. El área barrida se halló multiplicando la distancia recorrida en el tiempo efectivo de arrastre (10 minutos), por la abertura horizontal de la boca de la red (relinga superior de $7.7 \mathrm{~m}$ por $0.6 \mathrm{~m}$ de apertura; éste último corresponde al porcentaje teórico que asume una efectividad de apertura de la boca del $60 \%$ ) de acuerdo a Okonski y Martín (1977). Así se obtuvo la densidad por hectárea de las especies objeto de estudio (intraespecífica) y de todas las especies (interespecífica). La riqueza se estimó como el número total de especies registrado por arrastre. La profundidad, la temperatura y las coordenadas de los sitios de muestreo se obtuvieron a partir del SIBM del Invemar.

Para detectar tendencias de cambio en la talla de los individuos a nivel de especie, e inferir la influencia de las variables, se emplearon modelos aditivos generalizados (GAM, por sus siglas en inglés), con la talla como variable 
respuesta (las mediciones usadas en el análisis se indican en el cuadro 1) y como variables predictivas, profundidad, temperatura, densidad intraespecífica, densidad interespecífica, riqueza, latitud y longitud. Los GAM se realizaron por medio del programa R 2.12.2. Esta técnica se empleó ya que no requiere supuestos de estadística paramétrica, los cuales no se cumplieron para los datos de este estudio.

Un GAM es una extensión de los modelos lineales, pero permite que las funciones lineales de las variables predictivas sean remplazadas por funciones de "suavizamiento", y no necesita supuestos de las formas funcionales (Venables \& Dichmont, 2004) de modo que:

$$
y=\propto+\sum_{i=l}^{n} f i(X i)+\varepsilon
$$

Donde $y$ es la variable respuesta, $\mathrm{X}_{\mathrm{i}}$ son las variables predictivas, $\alpha$ es una constante y $\varepsilon$ es el error. Los $f i$ son estimados usando suavizadores. En este trabajo se utilizó un suavizador tipo spline (s) para estimar estas funciones no paramétricas. El procedimiento diagnóstico del GAM contempló el valor de significancia (p), el criterio de información Akaike (AIC) en la selección del mejor modelo y el porcentaje de devianza explicada por el modelo.

\section{RESULTADOS}

Astropecten alligator (Paxillosida: Astropectinidae): Se encontraron asociaciones significativas en todos los casos entre la talla de los ejemplares y las variables predictivas (Cuadro 1 y Cuadro 2). De acuerdo a los valores de devianza y del AIC, las variables que mejor explicaron los cambios en tamaño fueron la densidad intraespecífica y la temperatura (Cuadro 1 y Cuadro 2). Las dimensiones de $A$. alligator disminuyeron alrededor de una riqueza de 60 especies (Fig. 2). La talla disminuyó hasta una densidad intraespecífica cercana a 50 individuos por hectárea, luego aumentó y se mantuvo relativamente estable entre los $100 \mathrm{y}$ 150 individuos/ha (Fig. 3). Las tallas variaron con respecto a la densidad interespecífica, pero con tendencia general a disminuir con el aumento de la misma (Fig. 4). Hubo una inclinación al incremento en tallas con la profundidad (Fig. 5) y una tendencia de aumento con la disminución de la temperatura (Fig. 6). Los tamaños no mostraron grandes cambios

CUADRO 2

Resultados de la modelación GAM entre el tamaño de los ejemplares (mm) y las variables predictivas ambientales

TABLE 2

GAM results of the relationship between length $(\mathrm{mm})$ and biological predictor variables

\begin{tabular}{|c|c|c|c|c|c|c|c|c|}
\hline \multirow{2}{*}{ Especie } & \multicolumn{2}{|c|}{ Profundidad (m) } & \multicolumn{2}{|c|}{ Temperatura $\left({ }^{\circ} \mathrm{C}\right)$} & \multicolumn{2}{|c|}{ Latitud } & \multicolumn{2}{|c|}{ Longitud } \\
\hline & $\operatorname{Dev}(\%)$ & $\mathrm{P}$ & $\operatorname{Dev}(\%)$ & $\mathrm{P}$ & $\operatorname{Dev}(\%)$ & $\mathrm{P}$ & $\operatorname{Dev}(\%)$ & $\mathrm{P}$ \\
\hline Astropecten alligator & 7.483 & 0.000 & 8.601 & 0.000 & 2.772 & 0.000 & 6.400 & 0.000 \\
\hline Anasimus latus & 15.450 & 0.000 & 10.699 & 0.008 & 28.708 & 0.000 & 29.951 & 0.000 \\
\hline Brissopsis atlantica & 22.651 & 0.000 & 15.625 & 0.000 & 17.928 & 0.000 & 20.162 & 0.000 \\
\hline Brissopsis elongata & 24.651 & 0.000 & 44.086 & 0.000 & 70.700 & 0.000 & 63.414 & 0.000 \\
\hline Chasmocarcinus cylindricus & 44.387 & 0.034 & 52.972 & 0.212 & 37.378 & 0.000 & 23.043 & 0.000 \\
\hline Cosmioconcha nitens & 59.154 & 0.000 & 17.708 & 0.000 & 70.351 & 0.000 & 65.365 & 0.075 \\
\hline Eudolium crosseanum & 98.924 & 0.000 & 96.200 & 0.002 & 29.083 & 0.000 & 28.063 & 0.000 \\
\hline Nuculana acuta & 20.102 & 0.000 & 44.925 & 0.000 & 37.125 & 0.000 & 39.873 & 0.000 \\
\hline Achelous spinicarpus & 97.152 & 0.000 & 56.089 & 0.000 & 15.261 & 0.000 & 15.984 & 0.000 \\
\hline
\end{tabular}

$\mathrm{La}(\mathrm{s})$ celda(s) resaltada(s) es una de las dos (o las dos) que mostro mayor asociación con la variación de las tallas de las diferentes especies. Medidas tomadas a los individuos en el cuadro 1.

Cells in grey show the variable(s) that better explain the variation in length for each species. Measurements and acronyms as in table 1 . 

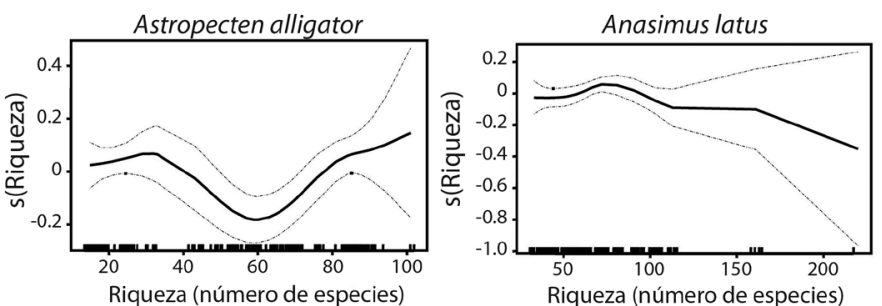

Brissopsis elongata
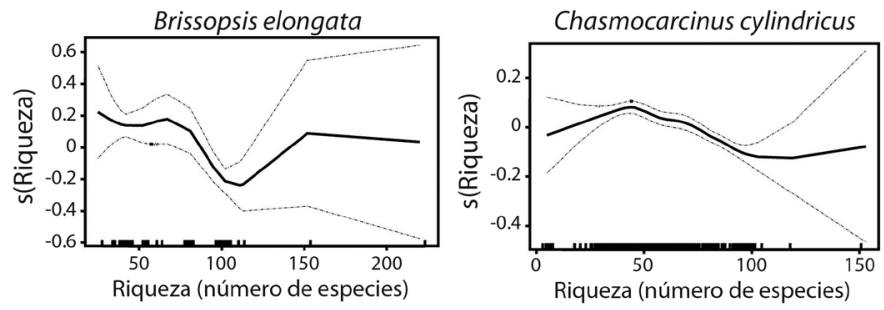

Eudolium crosseanum
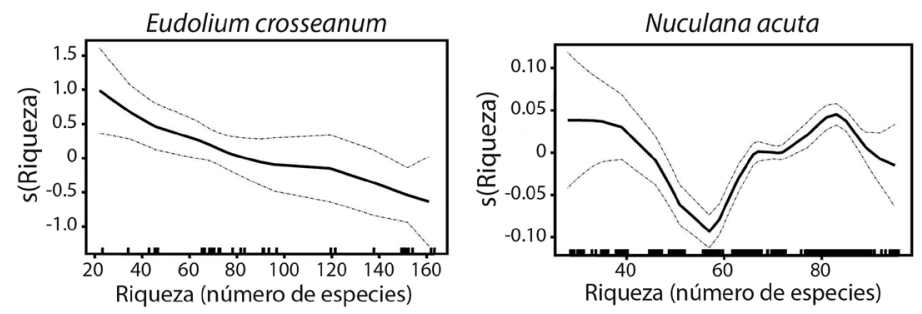

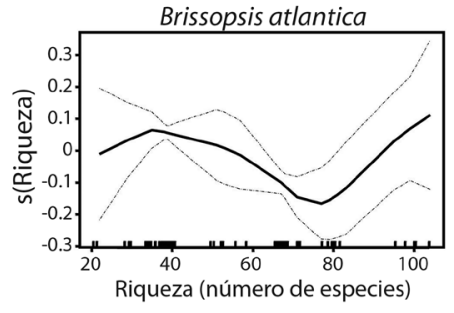

Cosmioconcha nitens

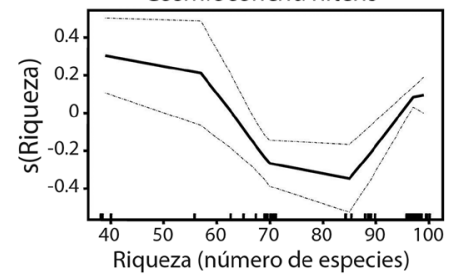

Achelous spinicarpus

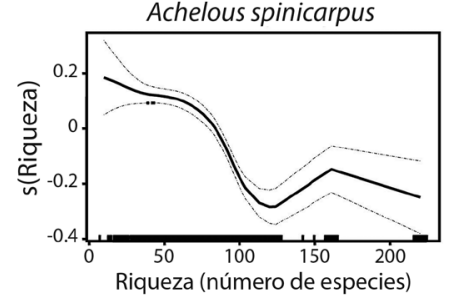

Fig. 2. Resultados de la modelación GAM de las relaciones funcionales entre la talla de las especies y la riqueza (número de especies) usando el suavizador tipo spine(s). Las líneas continuas indican el modelo ajustado y las punteadas son los intervalos de confianza (95\%).

Fig. 2. GAM model results with spline smoother (s), showing the functional relationships between body size and richness (number of species). The solid lines indicate the fitted model and the dashed lines are $95 \%$ confidence intervals.

en el gradiente latitudinal y los mayores valores se presentaron en los extremos del mismo; pero longitudinalmente, si se presentó una notoria disminución de la tallas entre $74^{\circ} \mathrm{W}$ y $73^{\circ} \mathrm{W}$ (Fig. 7 y Fig. 8).

\section{Anasimus latus (Decapoda: Inachoidi-} dae): Se presentaron relaciones significativas entre todas las variables predictivas y el tamaño de la especie, excepto por la densidad interespecífica (Cuadro 1 y Cuadro 2). De estas, las que mejor explicaron los cambios de talla fueron la latitud y la longitud (Cuadro 2). La talla de la especie parece mermar con el incremento en la riqueza de especies (Fig. 2). Los tamaños disminuyeron con respecto a la densidad intraespecífica entre 10 y 40 individuos/ha, $\mathrm{y}$ aumentaron nuevamente de 40 individuos/ ha en adelante (Fig. 3). Si bien no se presentó una relación significativa entre el tamaño de la especie y la densidad interespecífica (Cuadro 1), se observó una relación inversa entre ambos, ya que al aumentar el número de individuos/ha, el tamaño de los ejemplares tendió a disminuir (Fig. 4). Las dimensiones de A. latus tendieron a incrementarse con la profundidad, entre 50 y 300 m (Fig. 5) y con la disminución de la temperatura (Fig. 6). Con la latitud se observó un decrecimiento de los tamaños más o menos continuo (con algunos altibajos), desde antes de los $9^{\circ} \mathrm{N}$ hasta los $12^{\circ} \mathrm{N}$ (Fig. 7). Con la longitud se vio una disminución continua de las tallas entre $77^{\circ} \mathrm{W}$ y $74^{\circ} \mathrm{W}$, para luego aumentar de $\operatorname{los} 74^{\circ} \mathrm{W}$ a los $72^{\circ} \mathrm{W}$ (Fig. 8).

Brissopsis atlantica (Spatangoida: Brissidae): Todas las relaciones entre las variables predictivas y la talla de la especie resultaron 

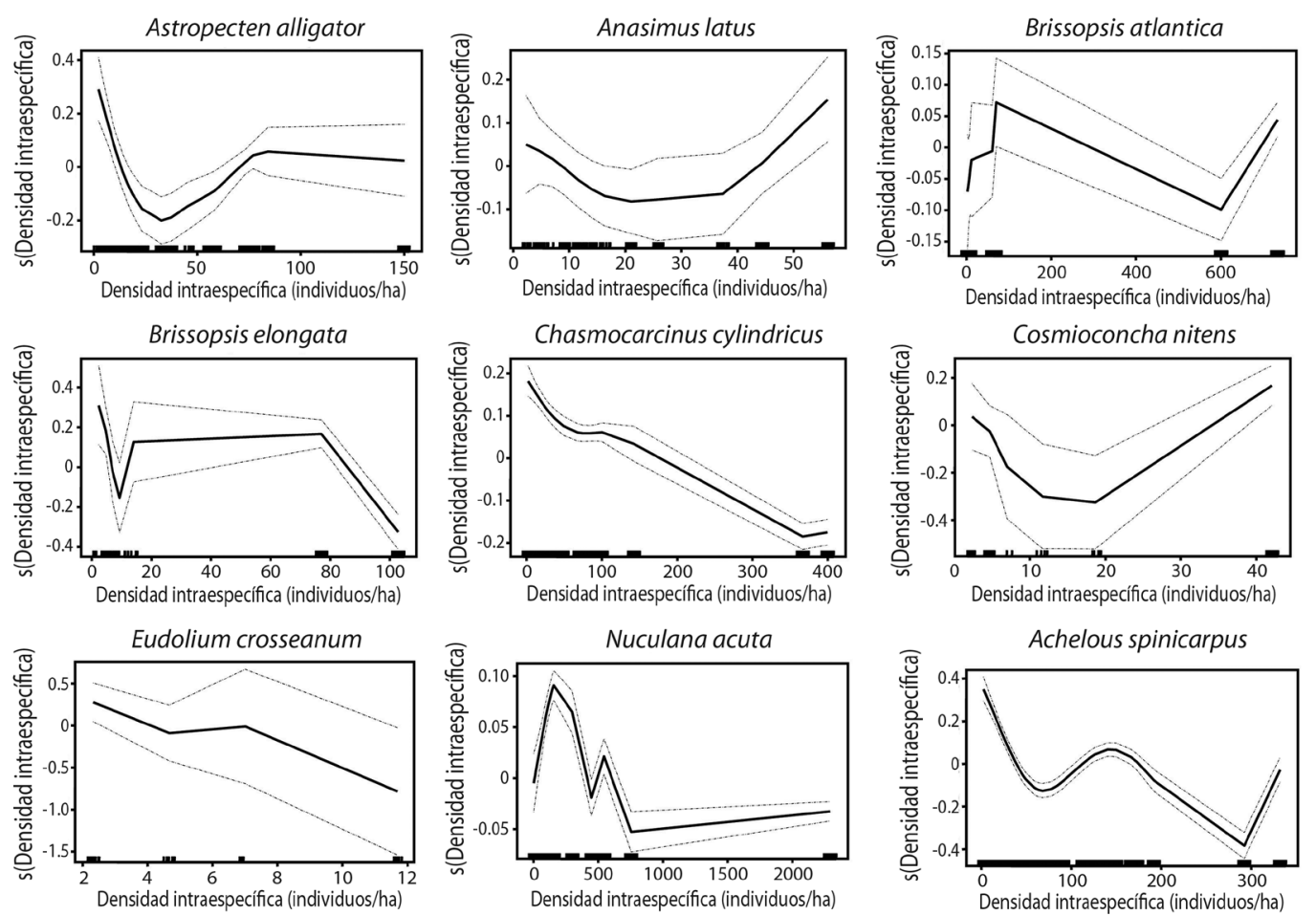

Fig. 3. Resultados de la modelación GAM de las relaciones funcionales entre la talla de las especies y la densidad intraespecífica usando el suavizador tipo spine(s). Las líneas continuas indican el modelo ajustado y las punteadas son los intervalos de confianza ( $95 \%$ ).

Fig. 3. GAM model results with spline smoother (s), showing the functional relationships between body size and intraspecific density. The solid lines indicate the fitted model and the dashed lines are $95 \%$ confidence intervals.

significativas (Cuadro 1 y Cuadro 2); los modelos que mejor explicaron las variaciones de tamaño de los ejemplares examinados fueron la densidad interespecífica y la profundidad (Cuadro 1 y Cuadro 2). La relación con la riqueza de especies tuvo un leve aumento de tallas de 20 a 40 especies, diminución de 40 a 80 especies y un nuevo incremento de tamaño de 80 a 100 especies (Fig. 2). En cuanto a la densidad intraespecífica, entre 0 y 100 individuos/ha se vio un incremento de dimensiones, después una disminución hasta 600 individuos/ha, para aumentar nuevamente después de los 600 individuos/ha (Fig. 3). Respecto a la densidad interespecífica, hubo una notoria disminución en las tallas de $B$. atlantica entre 0 y 1000 individuos/ha, luego aumentaron los tamaños entre 1000 y 2000 individuos/ha, y entre 2000 y 3000 individuos/ha se mantuvieron estables (Fig. 4). Con la profundidad se observó una disminución de las dimensiones de cero a $100 \mathrm{~m}$, luego un aumento de 100 a $300 \mathrm{~m}$ seguida de una disminución no tan pronunciada hasta los $500 \mathrm{~m}$, terminando en tamaños similares a los del inicio del gradiente batimétrico (Fig. 5). En términos generales, la relación entre las dimensiones de B. atlantica y la temperatura fue inversa (aumento de tamaños con la disminución de la temperatura), pero alrededor de los $25^{\circ} \mathrm{C}$ se dio un aumento de tallas (Fig. 6). En el gradiente latitudinal hubo una disminución de tamaño más o menos constante desde los $9^{\circ} \mathrm{N}$ hasta cerca de los $11^{\circ}$ $\mathrm{N}$, aumentando nuevamente hasta más de los $12^{\circ} \mathrm{N}$ (Fig. 7). Longitudinalmente, se observó una marcada disminución de los tamaños de $B$. atlantica entre $75^{\circ} \mathrm{W}$ y $74^{\circ} \mathrm{W}$ (Fig. 8). 

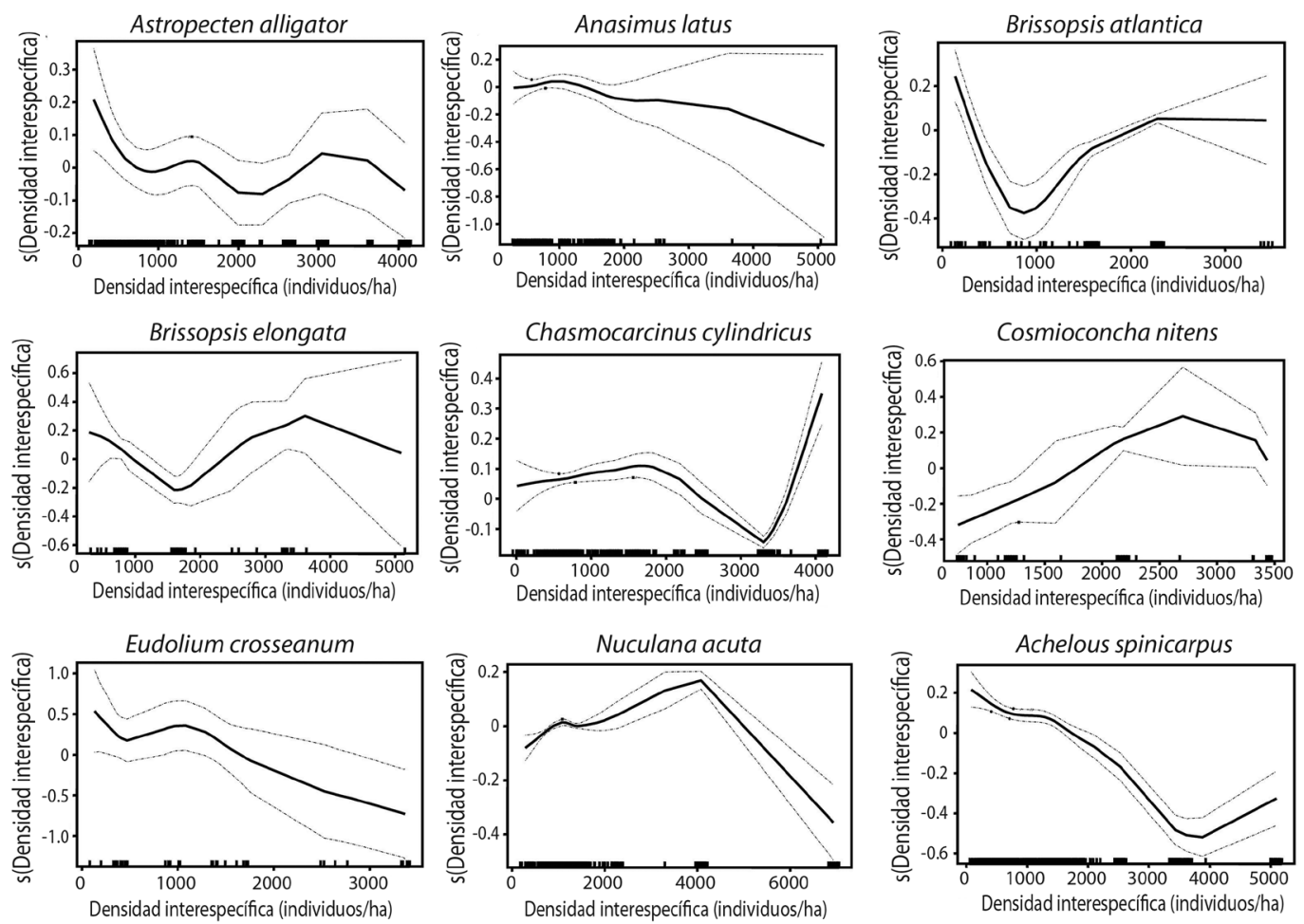

Fig. 4. Resultados de la modelación GAM de las relaciones funcionales entre la talla de las especies y la densidad interespecífica usando el suavizador tipo spine(s). Las líneas continuas indican el modelo ajustado y las punteadas son los intervalos de confianza (95\%).

Fig. 4. GAM model results with spline smoother (s), showing the functional relationships between body size and interspecific density. The solid lines indicate the fitted model and the dashed lines are $95 \%$ confidence intervals.

Brissopsis elongata (Spatangoida: Brissidae): Todas las asociaciones entre las variables predictivas y las dimensiones de esta especie fueron significativas (Cuadro 1 y Cuadro 2), y los modelos que mejor explicaron los resultados obtenidos fueron la densidad intraespecífica y la latitud (Cuadro 2). La relación entre la talla y la riqueza parece ser inversa (Fig. 2). Respecto a la densidad intraespecífica, se vio en términos generales una merma en los tamaños con el incremento de individuos/ ha, principalmente entre 80 y 100 individuos/ ha (Fig. 3). La relación entre la talla y la densidad interespecífica no fue clara (Fig. 4). Los tamaños de $B$. elongata mostraron tendencias generales de aumento con el incremento de profundidad y disminución de temperatura (Fig. 5 y Fig. 6). Se presentó una tendencia al aumento en la talla de los ejemplares en el gradiente latitudinal, pero con una disminución entre $11^{\circ} \mathrm{N}$ y $12^{\circ} \mathrm{N}$ (Fig. 7). Respecto a la longitud, las dimensiones de B. elongata tuvieron un incremento pronunciado desde menos de $76^{\circ} \mathrm{W}$ hasta $75^{\circ} \mathrm{W}$, y disminuyeron desde esta última hasta menos de $73^{\circ} \mathrm{W}$, para aumentar nuevamente después de $73^{\circ} \mathrm{W}$ (Fig. 8).

\section{Chasmocarcinus cylindricus (Decapoda:} Chasmocarcinidae): Las variables predictivas que presentaron una relación significativa con el comportamiento de los tamaños de la especie fueron: la profundidad, la densidad interespecífica, la riqueza (aunque con un valor marginal), la latitud y la longitud (Cuadro 1 y Cuadro 2). Las variables que mejor explicaron los cambios de talla fueron la temperatura 

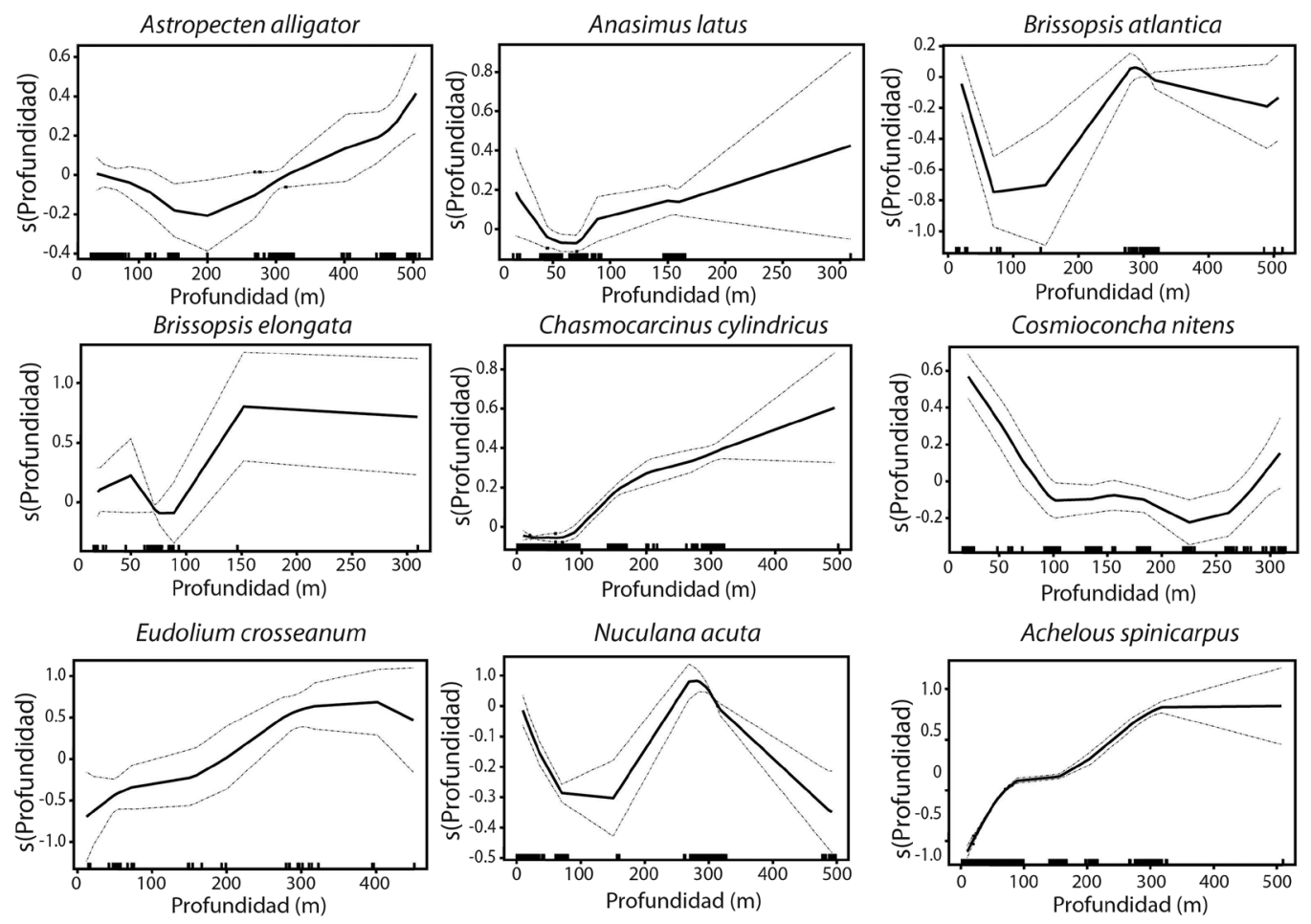

Fig. 5. Resultados de la modelación GAM de las relaciones funcionales entre la talla de las especies y la profundidad usando el suavizador tipo spine(s). Las líneas continuas indican el modelo ajustado y las punteadas son los intervalos de confianza $(95 \%)$.

Fig. 5. GAM model results with spline smoother (s), showing the functional relationships between body size and depth. The solid lines indicate the fitted model and the dashed lines are $95 \%$ confidence intervals.

y la profundidad (Cuadro 2). La asociación entre las tallas y la riqueza tuvo un aumento de 0 a 50 especies, seguida por una disminución hasta las 100 especies, donde hubo un leve incremento de las dimensiones hasta llegar a 150 especies (Fig. 2). La relación entre el tamaño de los organismos y la densidad intraespecífica fue inversa, visualizándose una disminución de las dimensiones de C. cylindricus al aumentar la cantidad de individuos/ha (Fig. 3). En cuanto a la densidad interespecífica, se vio un leve pero progresivo aumento entre $0 \mathrm{y}$ 2000 individuos/ha, luego disminuyó considerablemente después de los 3000 individuos/ha, para finalmente haber un abrupto aumento de tamaños hacia los 4000 individuos/ha (Fig. 4). Se presentó una clara tendencia de aumento de las tallas de los individuos con el incremento de la profundidad (Fig. 5) y la disminución temperatura (Fig. 6). Latitudinalmente se reflejó un detrimento en los tamaños de C. cylindricus aproximadamente entre $8^{\circ} \mathrm{N}$ y $9^{\circ} \mathrm{N}$, para después tener un notorio aumento hacia los $12^{\circ} \mathrm{N}$ (Fig. 7), resultado que es consecuente con el aumento de talla con la profundidad, ya que en el Caribe colombiano la latitud aumenta más o menos perpendicularmente con la línea de costa. Longitudinalmente, la talla de la especie disminuyó de $77^{\circ} \mathrm{W}$ a $75^{\circ} \mathrm{W}$, luego aumentó marcadamente de $75^{\circ} \mathrm{W}$ a $72^{\circ} \mathrm{W}$, y disminuyó nuevamente hasta los $71^{\circ} \mathrm{W}$ (Fig. 8).

\section{Cosmioconcha nitens (Neogastropoda:} Columbellidae): Esta especie presentó asociaciones significativas con todas las variables predictivas analizadas, excepto con la longitud (Cuadro 1 y Cuadro 2). De estas, las que mejor explicaron la variación de los tamaños hallados 

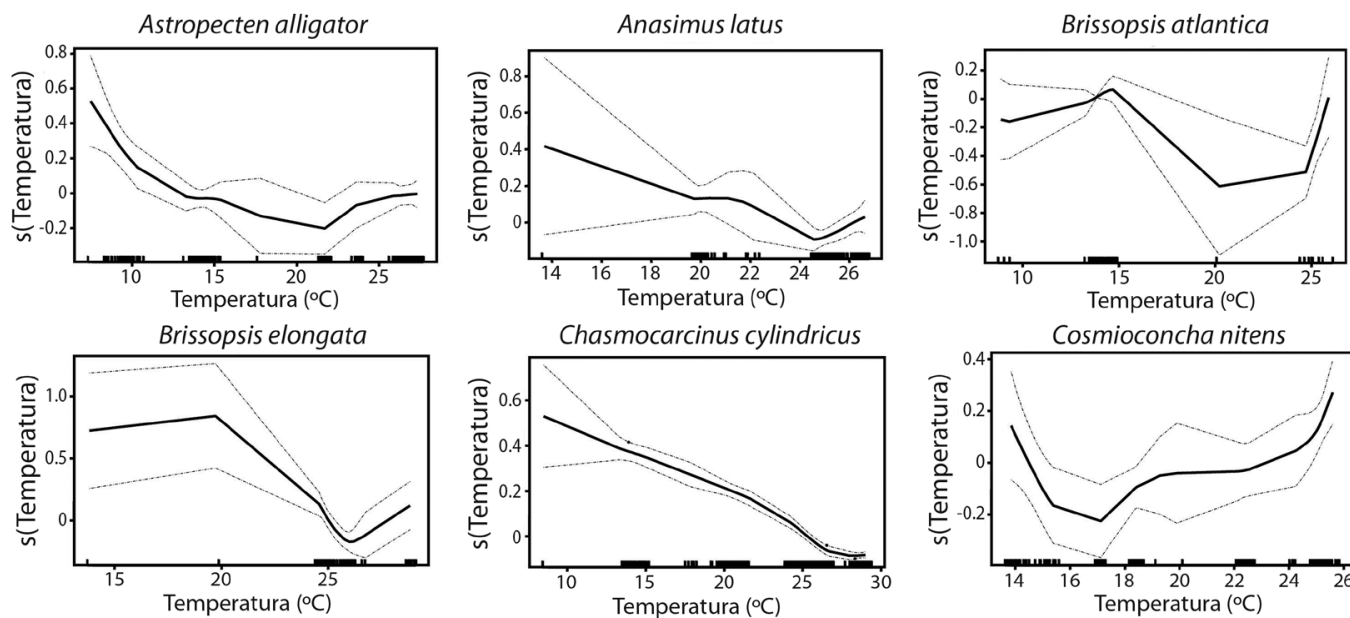

Chasmocarcinus cylindricus
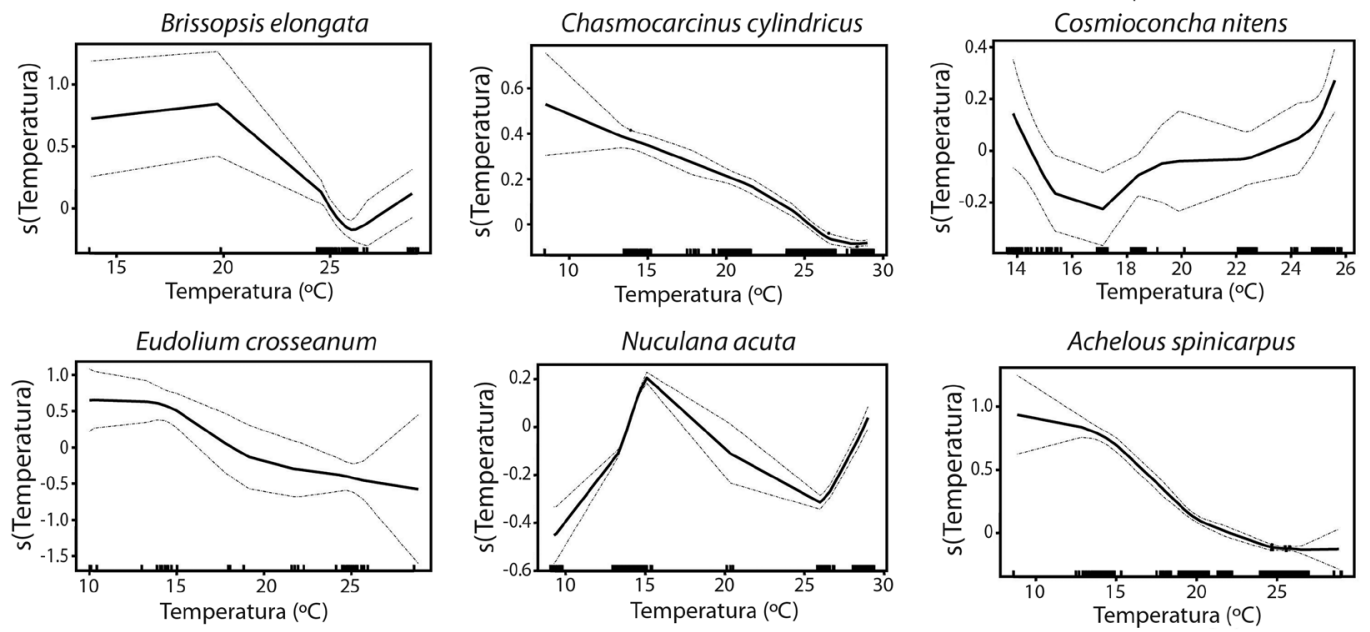

Fig. 6. Resultados de la modelación GAM de las relaciones funcionales entre la talla de las especies y la temperatura usando el suavizador tipo spine(s). Las líneas continuas indican el modelo ajustado y las punteadas son los intervalos de confianza (95\%).

Fig. 6. GAM model results with spline smoother (s), showing the functional relationships between body size and temperature.The solid lines indicate the fitted model and the dashed lines are $95 \%$ confidence intervals.

fueron la riqueza de especies y la densidad interespecífica (Cuadro 1). La primera tuvo una relación inversa con la talla de los ejemplares hasta las 80 especies, de 80 a 100 especies aumentaron las dimensiones nuevamente (Fig. 2). Con la segunda se observó un aumento de tamaños entre 1000 y 3000 individuos/ha y luego una disminución de 3000 a 3500 (individuos/ha, Fig. 4). Hubo una tendencia a la disminución de las dimensiones de $C$. nitens con la profundidad en casi todo el ámbito batimétrico abarcado (0 a 300 m, Fig. 5). Los tamaños decrecieron con la temperatura entre $14{ }^{\circ} \mathrm{C}$ y $18{ }^{\circ} \mathrm{C}$, y después aumentaron de $18{ }^{\circ} \mathrm{C}$ a $26^{\circ} \mathrm{C}$ (Fig. 6). La talla de la especie disminuyó con la densidad intraespecífica entre 0 y 20 individuos/ha; y de 20 a 40 individuos/ha aumentó (Fig. 3). Geográficamente, se observó un claro incremento de tamaños, latitudinalmente de $10^{\circ} \mathrm{N}$ a $12.5^{\circ} \mathrm{N}$, y longitudinalmente, de $76^{\circ} \mathrm{W}$ a $71^{\circ} \mathrm{W}$ (Fig. 7 y Fig. 8 ).

\section{Eudolium crosseanum (Littorini-} morpha: Tonnidae): Todas las variables predictivas tuvieron asociaciones significativas con los cambios en tamaño de E. crosseanum, con excepción de la densidad intraespecífica (Cuadro 1 y Cuadro 2). Las variables que mejor explicaron las variaciones de talla de la especie en el Caribe colombiano, fueron la profundidad y la temperatura (Cuadro 2). En cuanto a la asociación entre la riqueza de especies y el tamaño de los organismos, se vio una disminución de tallas con el incremento del número de especies (Fig. 2). Tanto la densidad intraespecífica como la interespecífica tuvieron una relación inversa con las dimensiones de E. crosseanum, ya que a medida que aumentaba el número 

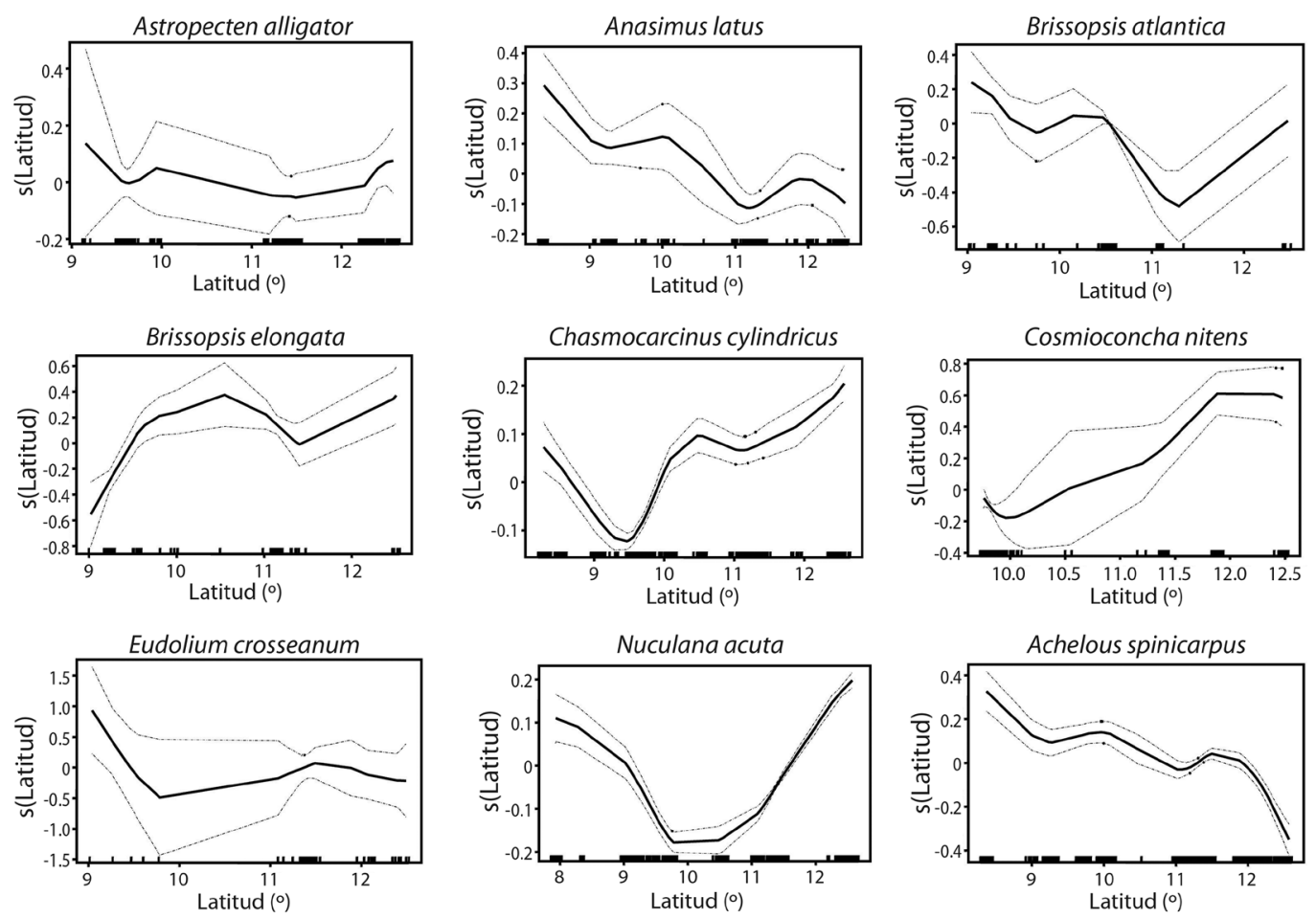

Fig. 7. Resultados de la modelación GAM de las relaciones funcionales entre la talla de las especies y la latitud usando el suavizador tipo spine(s). Las líneas continuas indican el modelo ajustado y las punteadas son los intervalos de confianza $(95 \%)$.

Fig. 7. GAM model results with spline smoother (s), showing the functional relationships between body size and latitude. The solid lines indicate the fitted model and the dashed lines are $95 \%$ confidence intervals.

de individuos por hectárea, disminuía la talla de los especímenes (Fig. 3 y Fig. 4). Se vio una clara tendencia al aumento del tamaño de la especie con la profundidad, y consecuentemente las mayores tallas se registraron en sitios con menores temperaturas (en un gradiente de $10^{\circ} \mathrm{C}$ a $25^{\circ} \mathrm{C}$, Fig. 5 y Fig. 6). Geográficamente, el tamaño de E. crosseanum disminuyó de $9^{\circ} \mathrm{N}$ a $10^{\circ} \mathrm{N}$ de latitud, luego aumentó de $10^{\circ} \mathrm{N}$ hasta cerca de los $12^{\circ} \mathrm{N}$, y disminuyó nuevamente (Fig. 7). Longitudinalmente, sus tallas no variaron considerablemente; las mayores dimensiones se encontraron antes de los $76^{\circ} \mathrm{W}$ y las menores después de los $72^{\circ} \mathrm{W}$ (Fig. 8).

\section{Nuculana acuta (Nuculida: Nuculidae):}

Las tallas de la especie se asociaron significativamente con todas las variables predictivas, y de estas, las variables que más explicaron las variaciones de tamaño fueron la temperatura y la longitud (Cuadro 1 y Cuadro 2). La relación entre la riqueza de especies y las dimensiones de este bivalvo indicaron una disminución de tamaños entre el rango de 40 y 60 especies (Fig. 2). La asociación entre la densidad intraespecífica y las tallas de $N$. acuta mostró una tendencia general de decrecimiento de tamaños con la abundancia de individuos/ha (Fig. 3). Con la densidad interespecífica se dio un incremento de tallas hasta los 4000 individuos/ha; de 4000 a 6000 individuos/ha los tamaños decrecieron hasta ser menores que las dimensiones registradas con los valores más bajos de densidad (Fig. 4). En el perfil batimétrico, se vio un decrecimiento de tamaños de cero a cerca de $200 \mathrm{~m}$, luego un aumento de 200 a $300 \mathrm{~m}$, terminado con una decrecimiento de 300 a 500 m (Fig. 5). En el gradiente de temperatura, 

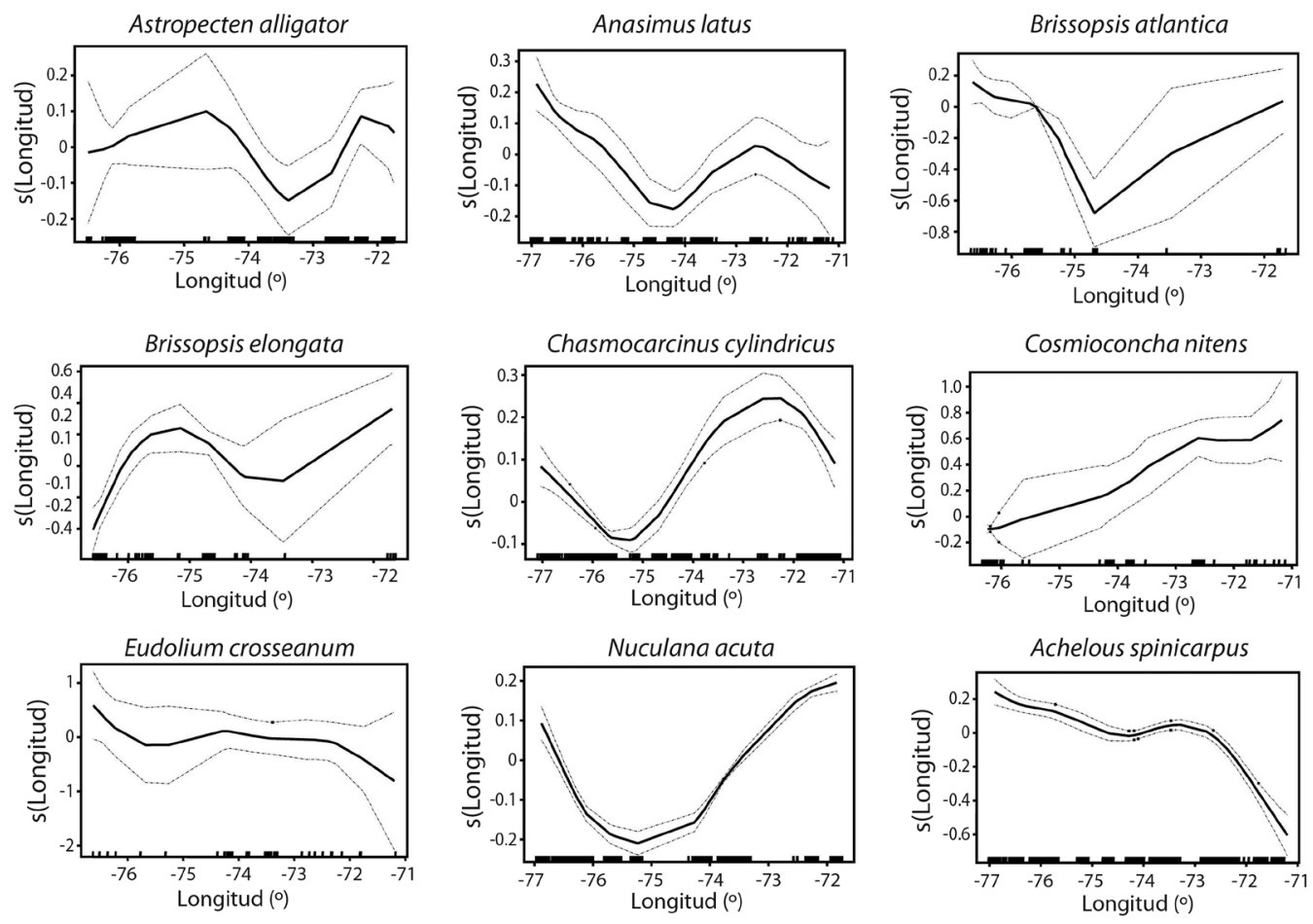

Fig. 8. Resultados de la modelación GAM de las relaciones funcionales entre la talla de las especies y la longitud usando el suavizador tipo spine(s). Las líneas continuas indican el modelo ajustado y las punteadas son los intervalos de confianza (95\%).

Fig. 8. GAM model results with spline smoother (s), showing the functional relationships between body size and longitude. The solid lines indicate the fitted model and the dashed lines are $95 \%$ confidence intervals.

se presentó un decrecimiento de tallas de $30^{\circ} \mathrm{C}$ a $25^{\circ} \mathrm{C}$, después un incremento de $25^{\circ} \mathrm{C}$ a 15 ${ }^{\circ} \mathrm{C}$ y finalmente, las dimensiones disminuyeron marcadamente de $15{ }^{\circ} \mathrm{C}$ a $10{ }^{\circ} \mathrm{C}$ (Fig. 6). Latitudinalmente, se evidenció una marcada disminución de tamaños hacia los $10^{\circ} \mathrm{N}$ (Fig. 7). Longitudinalmente, se observaron las menores dimensiones entre los $76^{\circ} \mathrm{W}$ y $74^{\circ} \mathrm{W}$ y un aumento después de $74^{\circ} \mathrm{W}$, hasta alcanzar las mayores tallas hacia los $72^{\circ} \mathrm{W}$ (Fig. 8).

\section{Achelous spinicarpus (Decapoda: Portu-}

nidae): Las siete variables predictivas empleadas en este estudio presentaron una relación significativa con los tamaños de la especie, y las variables que mejor explicaron las tendencias de cambio fueron la profundidad y la temperatura (Cuadro 1 y Cuadro 2). Las dimensiones de $A$. spinicarpus tendieron a disminuir con el incremento de la riqueza de especies (Fig. 2). En cuanto a las densidades intraespecífica e interespecífica, hubo una tendencia general a la disminución de los tamaños con el incremento del número de individuos/ha, pero las tallas aumentaron hacia los mayores valores en ambas variables (Fig. 3 y Fig. 4). Se encontró un claro aumento del tamaño de los ejemplares de $A$. spinicarpus en el perfil batimétrico (entre $0 \mathrm{y}$ 500 m, Fig. 5). En el gradiente térmico también se observa una notoria tendencia al aumento de las dimensiones de estos cangrejos de temperaturas altas a bajas (de $30{ }^{\circ} \mathrm{C}$ a $10{ }^{\circ} \mathrm{C}$, Fig. 6). Las variaciones de las tallas con respecto a la latitud y la longitud, indican una disminución paulatina de los tamaños del sur-oeste a nor-este del Caribe colombiano (Fig. 7 y Fig. 8). 


\section{DISCUSIÓN}

Los resultados demuestran que seis especies (Astropecten alligator, Anasimus latus, Brissopsis elongata, Chasmocarcinus cylindricus, Eudolium crosseanum, Achelous spinicarpus) incrementaron su tamaño corporal con el aumento de la profundidad del lecho marino y la disminución de la temperatura. Esto es contrario a lo que ha sido considerado como la característica más sobresaliente de los invertebrados (meiofauna, macrofauna y megafauna bentónica) en el ámbito batimétrico, que es la disminución en tamaño a medida que aumenta la profundidad (Thiel, 1975; Soetaert \& Heip, 1989; Gage \& Tyler, 1991; Warwick \& Clarke, 1996; Rex et al., 1999; Olabarria \& Thurson, 2003; Rex et al., 2006; Soetaert et al., 2009; van der Grient \& Rogers, 2015) y tampoco refleja lo planteado en el modelo teórico de talla óptima, que predice igualmente menor tamaño de los organismos con el incremento de profundidad (Sebens, 1982; 1987). Dicho modelo se basa en la interacción entre ingestión, costo y excedente. La talla óptima se alcanza al presentarse la mayor diferencia entre ingestión y costo, quedando así más energía para reproducción y/o crecimiento. Cuando toda la energía excedente es invertida en crecimiento, se alcanza la talla máxima. Los modelos de talla óptima para invertebrados marinos indican una disminución del tamaño corporal con la profundidad (Sebens, 1982; 1987) debido a la disminución de la oferta de alimento hacia zonas profundas (Sanders \& Hessler, 1969; Parsons et al., 1977; Lampitt et al., 1986; Hoey et al., 2004); esto reduciría la ingestión y aumentaría el costo de las funciones de alimentación con la profundidad, quedando menos energía para crecimiento.

Contrario a lo anterior, la disminución de alimento disponible con la profundidad podría inducir tamaños grandes, postulando que organismos de mayor talla son metabólicamente más eficientes por unidad de masa. Así, el costo energético para mantener una biomasa dada sería menor que el requerido por organismos pequeños para mantener la misma biomasa; por lo anterior, ejemplares de mayores dimensiones pueden maximizar el aprovechamiento de los escasos nutrientes en zonas profundas (Peters, 1983; Mahaut et al., 1995; Rex \& Etter, 1998; Rex et al., 1999). Acorde a lo mostrado para las seis especies mencionadas, los resultados aquí dan soporte a esta última hipótesis, aunque no se puede desconocer que en el ámbito batimétrico de este estudio $(10$ a $500 \mathrm{~m})$ los aportes continentales de nutrientes pueden ser importantes, como la contribución del río Magdalena y el transporte eólico de nutrientes del desierto al mar en La Guajira. Estos casos se discuten en detalle más adelante.

Aspectos adicionales que pueden promover tamaños grandes incluyen menor vulnerabilidad a la depredación y abarcar áreas más extensas para conseguir alimento accediendo a más ítems alimentarios, lo que les proporciona una ventaja competitiva sobre organismos más pequeños (Woodward et al., 2005). El aumento de masa corporal es una adaptación ante la depredación, especialmente importante para especies epifaunales de movimiento lento (Gage \& Tayler, 1991). Probablemente la tendencia al incremento de tallas de Astropecten alligator, Brissopsis elongata y Eudolium crosseanum esté relacionada con la estrategia adaptativa mencionada, ya que se caracterizan por su limitada movilidad (e.g., Zulliger \& Lessios, 2010).

La tendencia más marcada de incremento de talla con el aumento de profundidad y disminución de temperatura, fue exhibida por los crustáceos Chasmocarcinus cylindricus y Achelous spinicarpus. Algunos crustáceos oportunistas que consiguen alimento escarbando en el sedimento desarrollan grandes tamaños en zonas profundas, como una estrategia alimentaria relacionada con la necesidad de lograr alta movilidad para localizar fuentes de comida, que son más dispersas a mayores profundidades (Gage \& Tayler, 1991; Hoey et al., 2004). Hay que resaltar que, de todas las especies seleccionadas para este estudio, los crustáceos Brachyura se destacan por ser los organismos que cuentan con mayor capacidad de desplazamiento. 
De acuerdo a los resultados, la temperatura puede ejercer un efecto importante en la determinación de las dimensiones de los invertebrados estudiados, ya que para seis especies (Astropecten alligator, Anasimus latus, Brissopsis elongata, Chasmocarcinus cylindricus, Eudolium crosseanum y Achelous spinicarpus) fue una de las variables predictivas que mejor explicó los cambios de tamaño observados. La temperatura tiene un rol crucial en la determinación de las tallas de los invertebrados marinos (y de los organismos poiquilotermos en general), ya que la tasa metabólica varía con la temperatura. Así, un aumento de 10 ${ }^{\circ} \mathrm{C}$ dobla el "costo" metabólico (ley de Van Hoff), quedando menos energía disponible para que los individuos crezcan y se reproduzcan (Sebens, 1987). Teniendo en cuenta que todas las especies que mostraron un incremento de las dimensiones corporales con la disminución de la temperatura, se distribuyeron en ámbitos batimétricos que superaron $10{ }^{\circ} \mathrm{C}$ de diferencia entre zonas someras (alrededor de los $10 \mathrm{~m}$ de profundidad) y profundas (hasta $500 \mathrm{~m}$ ), es posible que el aumento de tamaños se deba (al menos parcialmente) a la merma del "costo" metabólico por la disminución de temperatura en zonas más profundas, quedando así más energía disponible para que estos organismos crezcan.

Dichos resultados son afines con los de otros estudios, ya que inclusive variaciones mínimas en temperatura entre hábitats, pueden propiciar notorias diferencias en los tamaños máximos observados, tasas de crecimiento y distribución de frecuencia de tallas de las poblaciones de invertebrados marinos (Atkinson \& Sibly, 1997). Se ha documentado que el tamaño de muchos poiquilotermos puede ser incrementado experimentalmente sometiendo a los ejemplares a bajas temperaturas. O'Dea, Rodriguez \& Romero (2007) estimaron que una disminución de tan solo $1{ }^{\circ} \mathrm{C}$, tanto en condiciones controladas como en el medio natural, puede causar un aumento del $5 \%$ del tamaño del briozoo Cupuladria exfragminis. En diferentes ambientes terrestres y marinos, se ha encontrado que los animales tienden a ser de mayor talla en los hábitats más fríos o en las partes más frías de su ámbito geográfico, probablemente como una adaptación para la retención de calor y conservación de la energía, patrón que ha sido llamado por algunos autores "la regla temperatura-talla" (en inglés the temperature size rule) (Gage \& Tayler, 1991; Atkinson, 1994; Angilletta \& Dunham, 2003).

A diferencia de las especies mencionadas hasta aquí, el molusco Cosmioconcha nitens exhibió una tendencia general hacia la disminución de la talla con la profundidad (hasta aproximadamente $250 \mathrm{~m}$, aunque de 250 a 300 $\mathrm{m}$ los tamaños aumentaron nuevamente), y no mostró un patrón de cambio de talla claro con la temperatura. Es posible que esta variación de tamaño esté relacionada con una estrategia alimentaria, ya que en otros moluscos como los del género Microgloma, la aparente tendencia hacia la miniaturización ha sido atribuida a una intensa selección natural para responder a la escasez de partículas de alimento en los sedimentos de mar profundo, permitiéndole al animal una selección más minuciosa de partículas de mayor valor nutricional. Debido a que estos individuos son macrófagos, tienen la capacidad de "evaluar" cada partícula potencial de alimento antes de ingerirla (Gage \& Tyler, 1991).

Las dos especies restantes, Brissopsis atlantica y Nuculana acuta no mostraron un patrón de variación de tamaño muy claro con respecto a los gradientes de temperatura y profundidad (aunque $B$. atlantica mostró cierta tendencia a aumentar su talla con el aumento de profundidad y disminución de temperatura). Este resultado puede deberse a que otras variables diferentes a la profundidad y la temperatura pueden estar influyendo en la determinación de la talla de estas especies. De hecho, la densidad interespecífica fue la variable que mejor explicó los cambios de dimensiones de $B$. atlantica, y la longitud resultó ser la segunda variable predictiva más importante para $N$. acuta. Las relaciones de las dimensiones corporales con dichas variables se discuten más adelante.

Cinco especies mostraron, en términos generales, una tendencia a la disminución 
de sus tamaños con el incremento de la densidad intraespecífica (Brissopsis atlantica, Chasmocarcinus cylindricus, Eudolium crosseanum, Achelous spinicarpus y Nuculana acuta), y tres (Anasimus latus, E. crosseanum y $A$. spinicarpus) con el aumento de la densidad interespecífica; en el resto de las especies no se vieron tendencias definidas con respecto a estas dos variables. En muchos ecosistemas terrestres y dulceacuícolas se ha registrado que con el aumento de la abundancia de individuos se encuentran más ejemplares pequeños que grandes (May, 1988; Reuman \& Cohen, 2004; Woodward et al., 2005). Generalmente, en zonas marinas someras, también se presenta una relación inversa entre talla y densidad. Por ejemplo, los asteroideos de aguas poco profundas alcanzan tamaños mucho más grandes en poblaciones con baja densidad, por una menor competencia por presas (Paine, 1976). Otro molusco, Acmaea scabra también presenta patrones de talla similares a los anteriores, con respecto a la densidad (Sutherland, 1970). Como los mencionados, existe un número importante de estudios sobre diferentes grupos de invertebrados marinos de aguas someras, en los que se registra una relación inversa entre la talla de los ejemplares y los niveles de densidad (Sebens, 1987).

A pesar de lo anterior, en los resultados de este trabajo no se ve reflejada contundentemente la relación inversa entre densidad y talla postulada, pero esto es consecuente con los hallazgos de estudios de mar profundo que indican que a diferencia de lo que se observa en zonas someras, hacia aguas profundas, la relación entre talla y densidad no es muy evidente, a pesar de que se presenta una notoria disminución de la densidad de individuos bentónicos con la profundidad (debido a la disminución en la oferta de alimento hacia zonas profundas) (Abele, 1974; Tietjen et al., 1989). Se ha observado que las tallas promedio de los individuos pueden decrecer, aumentar, o no cambiar, a pesar de la disminución paulatina de la abundancia de organismos en el perfil batimétrico (Rex \& Etter, 1998).
Tampoco se observó una relación muy clara entre la riqueza (número de especies) y las dimensiones corporales de las especies estudiadas. Tres de ellas (Anasimus latus, Eudolium croseanum y Achelous spinicarpus) mostraron una tendencia a la disminución del tamaño de los ejemplares con el incremento del número de especies acompañantes. La relación entre la talla y la riqueza ha sido más estudiada en ambientes terrestres que en marinos. Según May (1988), en general, los animales terrestres muestran una relación inversa entre la talla y la riqueza, principalmente por el agotamiento de recursos que se puede dar, al aumentar la cantidad de especies presentes en una misma área.

Por otra parte, Rex y Etter (1998) en su estudio sobre gasterópodos de mar profundo al oeste del Atlántico Norte, encontraron que el aumento en tamaño de los ejemplares con la profundidad, era acompañado por una disminución en la riqueza, y sugieren que la baja oferta de alimento en zonas profundas, no permite la coexistencia de muchas especies grandes. Lo anterior es consecuente con lo encontrado en el presente estudio, ya que las tres especies que mostraron una relación inversa entre la talla y la riqueza, también presentaron un aumento de envergadura corporal con el incremento de la profundidad, así que, probablemente, la disminución en la disponibilidad de nutrientes hacia zonas profundas impide que muchas especies grandes convivan. Además, la teoría alométrica indica que los organismos más grandes generan una mayor demanda energética total en el ambiente que los organismos pequeños (McMahon, 1973), y la hipótesis de "la riqueza de especies dependiente de la energía" dice que el número de especies que puede coexistir en una zona, está limitada por la disponibilidad de energía. No obstante, no se puede desconocer que otros factores que no se incluyeron en este estudio, como la heterogeneidad espacial, también pueden influir en la riqueza de especies (Tecchio et al., 2011).

Al analizar el comportamiento de las dimensiones corporales de las especies estudiadas en el ámbito geográfico, se hizo evidente una marcada disminución de tallas en la mitad 
del Caribe colombiano, que se observó principalmente en Astropecten alligator, Anasimus latus, Brissopsis atlantica, B. elongata, Chasmocarcinus cylindricus y Nuculana acuta. Esta disminución de tamaños se presentó específicamente hacia la desembocadura del río Magdalena. Este río produce grandes cambios físico-químicos en el ambiente marino, que pueden estar relacionados con las menores tallas de los invertebrados estudiados en su área de influencia.

El río Magdalena tiene un caudal promedio de $650 \mathrm{~m}^{3} \cdot \mathrm{s}^{-1}$, y el efecto de ese gran volumen de agua sobre la salinidad es importante (Márquez, 1982). Además cerca de la desembocadura, se presentan fuertes corrientes (turbiditas), inestabilidad del fondo y gran transporte de sedimentos (aproximadamente una tonelada por segundo) (Arcos-1, ISA \& $\mathrm{CIOH}, 2000)$, lo que puede impedir o limitar el establecimiento y normal desarrollo de algunas especies de invertebrados. La pluma del Magdalena acarrea aproximadamente 125 millones de $\mathrm{m}^{3}$ de sedimentos al año, razón por la cual, las aguas del sector permanecen turbias (Molina, Pelgrain, Suzunaga, \& Giraldo, 1996). A pesar de que el Magdalena es considerado una fuente de fertilización de las aguas marinas de la región (Blanco, 1988), debido a la turbidez que provoca, el área de influencia de su desembocadura es la zona con menos productividad primaria planctónica de toda la costa norte de Sur América (Márquez, 1982), y esta puede ser la principal causa de que las menores dimensiones corporales de las especies estudiadas, se hayan presentado en este sector, ya que normalmente, el tamaño alcanzado por los organismos bentónicos, depende de los nutrientes que son generados en la superficie por los productores primarios, y que luego son transportados hacia el fondo marino.

Por otra parte, hacia el norte de la desembocadura del río Magdalena se detectó un marcado aumento de tamaños $\left(74^{\circ}-71^{\circ} \mathrm{W}\right.$ y $\left.11^{\circ}-13^{\circ} \mathrm{N}\right)$ en siete de las nueve especies estudiadas (Astropecten alligator, Anasimus latus, Brissopsis atlantica, B. elongata, Chasmocarcinus cylindricus, Cosmioconcha nitens, Nuculana acuta), lo que puede estar relacionado con los eventos de surgencia que se presentan en la parte norte del Caribe colombiano. Con base en análisis históricos de corrientes, temperaturas superficiales y profundidades de la termoclina, se ha definido que el área de surgencia significativa se extiende desde Punta Gallinas (71 ${ }^{\circ} 39^{\prime} \mathrm{W}$ ), a lo largo de la costa hasta los $75^{\circ} \mathrm{W}$ (Bula, 1977, Andrade \& Barton 2005). En esta área, se produce el ascenso vertical de masas de aguas profundas, frías y ricas en nutrientes, propiciando una mayor productividad planctónica superficial (Álvarez-León et al., 1995). En diferentes áreas con eventos de surgencia alrededor del planeta, se ha documentado que la productividad superficial conduce a incrementar la abundancia y biomasa de los organismos bentónicos (Gage \& Tayler, 1991; Lavaleye, Duineveld, Berghuis, Kok, \& Witbaard, 2002). Este mismo comportamiento, se presenta en el norte del Caribe colombiano, ya que diferentes autores indican que la surgencia da lugar a capturas de más peso individual total, mayor talla y mejor estado de bienestar de los organismos capturados (Álvarez-León et al., 1995; CrialesHernández, García, \& Wolf, 2006).

De acuerdo con los resultados del presente estudio, los tamaños de los organismos bentónicos asociados a fondos sedimentarios en el Caribe colombiano, parecen ser determinados en gran medida por la disminución de la temperatura en el perfil batimétrico, posiblemente como una estrategia adaptativa para la maximización de la energía. Otros factores físicos y bióticos, tales como la influencia de fuertes descargas continentales, diferencias en productividad primaria entre áreas y eventos de surgencia, también parecen influenciar la talla de estos organismos, principalmente en zonas someras. Futuros estudios en ambientes tropicales podrían explorar cómo varía el tamaño del megabentos a mayores profundidades (más de $500 \mathrm{~m}$ ) donde la temperatura es relativamente invariable (Andrade \& Barton, 2005) y por ende otros factores pueden ser más determinantes.

La evidencia presentada en relación a la tendencia al aumento de tallas con la profundidad 
e inversa con la temperatura es contraria a lo indicado por los modelos teóricos de talla óptima que predicen un decrecimiento del tamaño de los organismos con el incremento de la profundidad, debido a la disminución de nutrientes hacia zonas profundas del océano. El aumento de tamaño observado en este estudio favorece más las hipótesis que postulan tamaños corporales grandes en la megafauna profunda como una estrategia adaptativa para la optimización del gasto energético, como ocurre con muchos organismos en las porciones más frías de su ámbito de distribución. Futuros estudios en el Caribe deberían examinar variaciones en la talla de la megafauna bentónica en zonas más profundas que las abarcadas por este estudio, donde la temperatura es más estable, por lo cual, otros factores pueden ser de mayor importancia para determinar el tamaño de los organismos.

\section{AGRADECIMIENTOS}

La realización de esta investigación fue posible gracias al apoyo del Instituto de Investigaciones Marinas, Invemar, Santa Marta, Colombia. Los autores agradecen en especial a los miembros del equipo de trabajo del Museo de Historia Natural Marina de Colombia del Invemar.

\section{RESUMEN}

Entender y predecir variaciones en el tamaño de los invertebrados megabentónicos sigue siendo un desafío importante en macroecología marina. Este estudio se realizó para identificar tendencias de cambio en la talla de la megafauna bentónica de fondos sedimentarios tropicales y conocer la influencia de variables que pueden determinar el tamaño de estos organismos, evaluando hipótesis y paradigmas ecológicos de mar profundo originados en zonas subtropicales y templadas. El área de estudio abarcó toda la plataforma continental del Caribe colombiano. Los ejemplares se recolectaron en 1998, 2001 y 2005 con red demersal semi-globo, entre 10 y $500 \mathrm{~m}$ de profundidad. Se seleccionaron las especies mejor representadas: Eudolium crosseanum, Cosmioconcha nitens, Nuculana acuta (moluscos), Astropecten alligator, Brissopsis atlantica, B. elongata (equinodermos), Anasimus latus, Chasmocarcinus cylindricus y Achelous spinicarpus (crustáceos). Para detectar tendencias significativas de cambio de tamaño, e inferir la influencia de variables bióticas y ambientales, se emplearon modelos aditivos generalizados, donde la talla fue la variable respuesta y las variables predictivas fueron: profundidad, temperatura, densidad intraespecífica e interespecífica, riqueza, latitud y longitud. Se midieron 7000 ejemplares en total. Seis especies presentaron un incremento de la talla con el aumento de la profundidad y la disminución de la temperatura. Dichas especies abarcaron ámbitos batimétricos que superaron $\operatorname{los} 10^{\circ} \mathrm{C}$ de diferencia entre zonas someras y profundas. Geográficamente se presentó una marcada disminución de tallas en el área de influencia de la desembocadura del río Magdalena, debida posiblemente a los fuertes cambios físicosquímicos que este río genera, principalmente porque es la zona con menos productividad primaria planctónica de toda el área de estudio. Hacia el norte de la desembocadura del río Magdalena se presentó un notorio aumento de tamaños entre $\operatorname{los}\left(74^{\circ}-71^{\circ} \mathrm{W}\right.$ y $\left.11^{\circ}-13^{\circ} \mathrm{N}\right)$, que puede deberse a la surgencia que se presenta en el norte del Caribe colombiano. La relación entre la densidad de individuos y la talla no fue clara. Sin embargo cinco especies mostraron una relación inversa con la densidad intraespecífica, y tres con la interespecífica. Temperatura y profundidad fueron las variables que mejor explicaron los cambios de talla detectados. La mayoría de especies presentaron un aumento de dimensiones corporales con la disminución de la temperatura en el perfil batimétrico. La tendencia al aumento de tallas con la profundidad es contraria a lo indicado por los modelos teóricos de talla óptima (pero acorde con algunos estudios más recientes), que predicen un decrecimiento del tamaño de los organismos con el incremento de la profundidad, debido a la disminución de nutrientes hacia zonas profundas del océano. Es posible que el aumento de tamaño sea una estrategia adaptativa para la maximización de la energía, como ocurre con muchos organismos en las porciones más frías de su ámbito de distribución. Futuros estudios en el Caribe deberían examinar variaciones en la talla de la megafauna bentónica en zonas más profundas (más de $500 \mathrm{~m}$ ), donde la temperatura varía menos, razón por la cual otros factores pueden ser más importantes para determinar el tamaño de estos organismos.

Palabras clave: talla, variables predictivas, megafauna bentónica, fondos blandos tropicales, Caribe colombiano.

\section{REFERENCIAS}

Abele, L. G. (1974). Species diversity of decapod crustaceans in marine habitats. Ecology, 55(1), 156-161. doi.org/10.2307/1934629

Álvarez-León, L., Aguilera-Quiñones, J., Andrade-Amaya, C. \& Novak, P. (1995). Caracterización general de la zona de surgencia en La Guajira colombiana. Revista de la Academia Colombiana de Ciencias Físicas y Naturales, 19(75), 679-694. 
Andrade, C., \& Barton, E. (2005). The Guajira upwelling system. Continental Shelf Research, 25, 1003-1022. doi:10.1016/j.csr.2004.12.012

Angilletta, M., \& Dunham, A. (2003). The temperaturesize rule in ectotherms: simple evolutionary explanations may not be general. American Naturalist, $162(3), 332-342$.

Arcos-1, ISA, \& CIOH. (2000). Estudio de impacto ambiental del proyecto de cable submarino de fibra óptica ARCOS-1. EIA. Informe técnico. CIHO, Cartagena, Colombia.

Atkinson, D. (1994). Temperature and organism sizea biological law for ectotherms. Advances in Ecological Research, 25, 1-58. doi:10.1016/ S0065-2504(08)60212-3

Atkinson, D., \& Sibly, R. (1997). Why are organisms usually bigger in colder environments? Making sense of a life history puzzle. Trends in Ecology and Evolution, 12, 235-239. doi:10.1016/S0169-5347(97)01058-6

Berkenbusch, K., Probert, P. K., \& Nodder, S. D. (2011). Comparative biomass of sediment benthos across a depth transect, Chatham Rise, Southwest Pacific Ocean. Marine Ecology Progress Series, 425, 79-90.

Blackburn, T., \& Gaston, K. (1994). Animal body size distributions: patterns, mechanisms and implications. Trends in Ecology and Evolution, 9, 471-474. doi: 10.1016/0169-5347(94)90311-5

Blanco, J. (1988). Las variaciones ambientales estacionales en las aguas costeras y su importancia para la pesca en la región de Santa Marta, Caribe colombiano (Tesis de Maestría). Universidad Nacional de Colombia, Bogotá.

Bula, G. (1977). Algas marinas bénticas indicadoras de un área afectada por aguas de surgencia frente a la costa Caribe de Colombia. Anales del Instituto de Investigaciones Marinas Punta Betín, 9, 45-71.

Childress, J. J., \& Thuesen, E. V. (1993). Effects of hydrostatic pressure on metabolic rates of six species of deep-sea gelatinous zooplankton. Limnology and Oceanography, 38(3), 665-670. doi: 10.4319/ lo.1993.38.3.0665

Criales-Hernández, I. M., García, C. B., \& Wolf, M. (2006). Flujos de biomasa y estructura de un ecosistema de surgencia tropical en La Guajira, Caribe colombiano. Revista de Biología Tropical, 54(4), 1257-1282.

Danovaro, R., Snelgrove, P. V., \& Tyler, P. (2014). Challenging the paradigms of deep-sea ecology. Trends in Ecology \& Evolution, 29(8), 465-475. doi. org/10.1016/j.tree.2014.06.002

Durden, J. M., Bett, B. J., Jones, D. O., Huvenne, V. A., \& Ruhl, H. A. (2015). Abyssal hills - hidden source of increased habitat heterogeneity, benthic megafaunal biomass and diversity in the deep sea. Progress in Oceanography, 137, 209-218.

Escobar-Briones, E., \& Alvarez, F. (2002). Modern approaches to the study of Crustacea. New York: Springer Science.

Fierro, M. A. (2004). Estudio de los principales aspectos biológicos y de distribución de las especies dominantes de Agononida, Munida y Munidopsis en el Caribe colombiano (Crustacea: Decapoda: Galatheidae) (Tesis de pregrado). Universidad Nacional de Colombia, Bogotá.

Gage, J. D., \& Tyler, P. A. (1991). Deep-sea biology: a natural history of organisms at the deep-sea floor. Cambridge: Cambridge University Press.

Hardy, S. M., Smith, C. R., \& Thurnherr, A. M. (2015). Can the source-sink hypothesis explain macrofaunal abundance patterns in the abyss? A modelling test. Proceedings of the Royal Society B, 282, 20150193.

Hoey, G. V., Degraer, S., \& Vincx, M. (2004). Macrobenthic community structure of soft-bottom sediments at the Belgian continental shelf. Estuarine, Costal and Shelf Science, 59, 599-613. doi:10.1016/j. ecss.2003.11.005

Instituto de Investigaciones Marinas y Costeras (Invemar) (2000). Informe final del proyecto caracterización de la macrofauna del Caribe colombiano. Fase 1: Epifauna de la franja superior del talud continental $(150-450 \mathrm{~m})$. Santa Marta: Instituto de Investigaciones Marinas y Costeras (Invemar).

Instituto de Investigaciones Marinas y Costeras (Invemar). (2002a). Informe del estado de los ambientes marinos y costeros de Colombia: Año 2002. Serie de publicaciones periódicas No. 8. Santa Marta: Instituto de Investigaciones Marinas y Costeras (Invemar).

Invemar. (2002b). Informe final del proyecto caracterización y catalogación de la macrofauna marina del Caribe colombiano, Fase 2. Santa Marta: Instituto de Investigaciones Marinas y Costeras (Invemar).

Invemar \& Corpoguajira. (2006). Informe final del proyecto caracterización de la zona costera del departamento de La Guajira: una aproximación para su manejo integrado. Santa Marta: Instituto de Investigaciones Marinas y Costeras (Invemar).

Jensen, P. (1988). Nematode assemblages in the deep-sea benthos of the Norwegian Sea. Deep-Sea Research, $35,1173-1184$

Lampitt, R. S., Billett, D. S., \& Rice, A. L. (1986). Biomass of the invertebrate megabenthos from 500 to $4100 \mathrm{~m}$ in the northeast Atlantic Ocean. Marine Biology, 93, 69-81. doi: 10.1007/BF00428656

Lavaleye, M. S., Duineveld, G. C., Berghuis, E. M., Kok, A., \& Witbaard, R. A. (2002). Comparison between 
the megafauna communities on the N.W. Iberian and Celtic continental margins-effects of coastal upwelling? Progress in Oceanography, 52, 459-476. doi:10.1016/S0079-6611(02)00019-8

Leduc, D., Pilditch, C. A., \& Nodder, S. D. (2016). Partitioning the contributions of mega-, macroand meiofauna to benthic metabolism on the upper continental slope of New Zealand: Potential links with environmental factor sand trawling intensity. Deep-Sea Research I, 108, 1-12. doi.org/10.1016/j. dsr.2015.12.003

Mahaut, M. L., Sibuet, M., \& Shirayama, Y. (1995). Weight-dependent respiration rates in deep-sea organisms. Deep-Sea Research, 42, 1575-1582. doi: 10.1016/0967-0637(95)00070-M

Marcus, N. H. (1983). Phenotypic variability in echinoderms. En M. Jangoux \& J. M. Lawrence (Eds.), Echinoderm Studies (pp. 18-32). Rotterdam: A. A. Balkema.

Márquez, G. (1982). Los sistemas ecológicos marinos del sector adyacente a Santa Marta, Caribe colombiano I: generalidades. Ecología Tropical, 2(1), 5-13.

May, R. M. (1988). How many species are there on the earth? Science, 241 (4872), 1441-1449. doi: $10.2307 / 1702670$

Mcclain, C. R., Rex, M. A., \& Etter, R. J. (2009). Patterns in deep-sea macroecology. En J. D. Witman \& R. Kaustuv (Eds), Marine macroecology (pp. 65-100). Londres: The University of Chicago Press.

McMahon, T. (1973). Size and shape in biology. Science, 179, 1201-1204. doi: 10.2307/1735749

Molina, A., Pelgrain, A., Suzunaga, J., \& Giraldo, L. (1996). Comportamiento de la dinámica marina en el sector costero entre Galerazamba y Cartagena. Boletín Cientifico CIOH, 17, 73-78.

O’Dea, A., Rodríguez, F., \& Romero, T. (2007). Response of zooid size in Cupuladria exfragminis (Bryozoa) to simulated upwelling temperatures. Marine Ecology, 28, 1-9. doi:10.1111/j.1439-0485.2006.00144.x

Okonski, S. L., \& Martin, L. W. (1977). Materiales didácticos para la capacitación en tecnología de artes y métodos de pesca. Contribución al estudio de las pesquerías de México. México: Food and Agriculture Organization (FAO).

Olabarria, C., \& Thurston, M. H. (2003). Latitudinal and bathymetric trends in body size of the deep-sea gastropod (King) Troschelia berniciensis. Marine Biology, 143, 723-730. doi: 10.1007/s00227-003-1116-6

Paine, R. T. (1976). Size-limited predation: an observational and experimental approach with the Pisaster-Mytilus interaction. Ecology, 57, 858-73. doi. org/10.2307/1941053
Parsons, T. R., Takahashi, M., \& Hargrave, B. (1977). Biological Oceanographic Processes. Oxford: Pergamon Press.

Peters, R. H. (1983). The ecological implications of body size. Cambridge: Cambridge University Press.

Pfannkuche, O. (1985). The deep-sea meiofauna of the Porcupine Seabright and abyssal plain (N.E. Atlantic): population structure, distribution, standing stocks. Oceanologica Acta, 8, 343-353.

Polloni, P., Haedrich, R., Rowe, G., \& Clifford, C. H. (1979). The size-depth relationship in deep ocean animals. Internationale Reveu der Gesamten Hydrobiologie, 64, 39-46. doi: 10.1002/iroh.19790640103

Reuman, D. C., \& Cohen, J. E. (2004). Trophic links' length and slope in the Tuesday Lake food web with species' body mass and numerical abundance. Journal of Animal Ecology, 73, 852-866. doi: 10.1111/j.0021-8790.2004.00856.x

Rex, M. A., \& Etter, R. J. (1998). Bathymetric patterns of body size: implications for deep-sea biodiversity. Deep-Sea Research II, 45, 103-127.

Rex, M. A., Etter, R. J., Clain, A. J., \& Hill, M. S. (1999). Bathymetric patterns of body size in deepsea gastropods. Evolution, 53(4), 1298-1301. doi: $10.2307 / 2640833$

Rex, M. A., Etter, R. J., Morris, J. S., Crouse, J., McClain, J. C., Johnson, N. A., ... \& Avery, R. (2006). Global bathymetric patterns of standing stock and body size in the deep-sea benthos. Marine Ecology Progress Series, 317, 1-8.

Sanders, H. L., \& Hessler, R. (1969). Ecology of the deepsea benthos. Science, 163, 1419-1424. doi: 10.1126/ science.163.3874.1419

Sebens, K. P. (1982). The limits to indeterminate growth: An optimal size model applied to passive suspension feeders. Ecology, 63, 209-222. doi.org/10.2307/1937045

Sebens, K. P. (1987). The ecology of indeterminate growth in animals. Annual Review of Ecology Evolution and Systematics, 18, 371-407.

Soetaert, K., \& Heip, C. (1989). The size of nematode assemblages along a Mediterranean deepsea transect. Deep-Sea Research, 36A, 93-102. doi:10.1016/0198-0149(89)90020-4

Soetaert, K., Franco, M., Lampadaríou, N., Muthumbi, A., Steyaert, M., Vandepitte L., ... \& Vanaverbeke, J. (2009). Factors affecting nematode biomass, length and width from the shelf to the deep sea. Marine Ecology Progress Series, 392, 123-132. doi: 10.3354/ meps08202

Sturm, C. F., Pearce, T. A., \& Valdés, A. (2007). The Mollusks: a guide to their study, collection, and 
preservation. Middletown: American Library Association Choice.

Sutherland, J. P. (1970). Dynamics of high and low populations of the limpet, Acmaea scabra (Gould). Ecological Monographs, 40, 169-188. doi. org/10.2307/1942294

Tecchio, S., Ramírez-Llodra, E., Sardà, F., Company, J. B., Palomera, I., Mechó, A., Pedrosa-Pàmies, R., \& Sanchez-Vidal, A. (2011). Drivers of deep Mediterranean megabenthos communities along longitudinal and bathymetric gradients. Marine Ecology Progress Series, 439, 181-192. doi: 10.3354/meps09333

Thiel, H. (1975). The size structure of the deep-sea benthos. Internationale Reveu der Gesamten Hydrobiologie, 60, 575-606.

Tietjen, J. H., Deming, J. W., Rowe, G. T., Macko, S., \& Wilke, R. J. (1989). Meiobenthos of the Hatteras abyssal plain and Puerto Rico Trench: abundance, biomass and associations with bacteria and particulate fluxes. Deep-Sea Research, 18, 941-57. doi:10.1016/0198-0149(89)90058-7

Van der Grient, J. M., \& Rogers, A. D. (2015). Body size versus depth: regional and taxonomical variation in deep-sea meio- and macrofaunal organisms. Advances in Marine Biology, 71, 71-99. doi.org/10.1016/ bs.amb.2015.07.002
Venables, W. N., \& Dichmont, C. M. (2004). GLMs, GAMs and GLMMs: an overview of theory for applications in fisheries research. Fisheries Research, 70, 319-337. doi:10.1016/j.fishres.2004.08.011

Warwick, R. M., \& Clarke, K. R. (1996). Relationships between body-size, species abundance and diversity in marine benthic assemblages: facts or artefacts? Journal of Experimental Marine Biology and Ecology, 202, 63-71. doi:10.1016/0022-0981(96)00031-7

Webb, T. J. (2012). Marine and terrestrial ecology: unifying concepts, revealing differences. Trends in Ecology and Evolution, 27(10) 535-541. doi.org/10.1016/j. tree.2012.06.002

Woodward, G., Ebenman, B., Emmerson, M., Montoya, J. M., Olesen, J. M., Valido, A., \& Warren, P. H. (2005). Body size in ecological networks. Trends in Ecology and Evolution, 20(7), 1-8. doi.org/10.1016/j. tree.2005.04.005

Zulliger, D., \& Lessios, H. A. (2010). Phylogenetic relationships in the genus Astropecten Gray (Paxillosida: Astropectinidae) on a global scale: molecular evidence for morphological convergence, speciescomplexes and possible cryptic speciation. Zootaxa, 2504, 1-19. 